\title{
Effective magnetic permeability of compacted metal powders at microwave frequencies
}

\author{
I.I. Volkovskaya $^{1,2}$, V.E. Semenov ${ }^{1}$, and K.I. Rybakov ${ }^{1,2}$ \\ ${ }^{1}$ Institute of Applied Physics, Russian Academy of Sciences, Nizhny Novgorod, Russia, volkovskaya.ira@mail.ru \\ ${ }^{2}$ Lobachevsky State University, Nizhny Novgorod, Russia
}

The calculation of the absorption of electromagnetic waves in an inhomogeneous medium consisting of many particles relies on the effective values of the dielectric permittivity and the magnetic permeability of the medium [1]. The subject of this paper is the effective magnetic permeability of an inhomogeneous medium consisting of metal particles. The response of a medium consisting of metal particles when exposed to a high-frequency magnetic field is determined by the induced magnetic moments associated with the excitation of eddy electric currents in the particles.

Until now, no method for calculating the magnetictype losses has been proposed that adequately takes into account the mutual influence of particles when their volume concentration is significant (for example, during the sintering process). If the relative volume concentra-tion of conductive particles in a medium, $\eta$ (defined as the ratio of volume of the particulate material to the total volume of the medium), is so small that each particle can be considered isolated, the effective permeability of the medium may be defined as

$$
\mu_{\mathrm{eff}}=1+4 \pi \eta \alpha_{m}
$$

where $\alpha_{m}$ is the complex magnetic polarizability of a spherical metal particle of radius $a$ placed in an alternating magnetic field in vacuum [2]:

$$
\alpha_{m}=-\frac{3}{8 \pi}\left[1-\frac{3}{(k a)^{2}}+\frac{3}{k a \tan (k a)}\right],
$$

where $k=\omega \sqrt{\varepsilon} / c \approx(1+i) / \delta, \varepsilon=\varepsilon^{\prime}+i 4 \pi \sigma / \omega, \sigma$ is the conductivity of the particle material, $\omega$ is the cyclic frequency of magnetic field oscillation, $\delta=c / \sqrt{2 \pi \sigma \omega}$ is the penetration depth of the magnetic field into metal (skin depth), $c$ is the speed of light, and $i$ is the imaginary unit.

To account for the mutual influence of particles at high values of the relative volume concentration, it is necessary to determine the magnetic dipole moment of a particle in a uniform alternating magnetic field $\mathbf{H}_{0}$ when the particle is surrounded by an effective medium with a magnetic permeability $\mu_{\text {eff. }}$ Similarly to [2], the magnetic field outside the spherical particle is a superposition of the external field and the dipolar field created by the particle:

$$
\mathbf{H}=\mathbf{H}_{0}-A \nabla\left(\frac{\mathbf{H}_{0} \cdot \mathbf{r}}{r^{3}}\right),
$$

where $A$ is an arbitrary constant that is related to the magnetic dipole moment of the particle, $\mathbf{p}_{m}$, as

$$
\mathbf{p}_{m}=\left(\frac{1+2 \mu_{\mathrm{eff}}}{3} A+\frac{\mu_{\mathrm{eff}}-1}{3} a^{3}\right) \mathbf{H}_{0} .
$$

The magnetic field inside the conductive particle can be represented as

$$
\mathbf{H}=B \operatorname{rot} \operatorname{rot}\left[\frac{\sin (k r)}{r} \mathbf{H}_{0}\right]
$$

where $B$ is an arbitrary constant, $k=\omega \sqrt{\varepsilon \mu} / c, \varepsilon$ and $\mu$ are the dielectric permittivity and magnetic permeability of the material of the particle. $A$ and $B$ can be obtained from the conditions at the boundary between the spherical particle and the effective medium that surrounds it. This determines the magnetic dipole moment of the particle (4):

$$
\mathbf{p}_{m}=-\frac{a^{3}}{2} \cdot \frac{\mu_{\mathrm{eff}}\left[\xi(1+2 \mu)+(k a)^{2}\right]}{\xi\left(\mu_{\mathrm{eff}}-\mu\right)+(k a)^{2} \mu_{\mathrm{eff}}} \mathbf{H}_{\infty},
$$

where $\xi=k a / \tan (k a)-1$.

The effective medium can be represented as a medium filled with metallic spherical particles with magnetic dipole moments $\mathbf{p}_{m j}$ and void space. The sum of all the dipole moments of metallic spherical inclusions in the volume of the effective medium $V$ is equal to the total dipole moment of this volume $\mathbf{p}_{m \text { eff }}$ :

$$
\mathbf{p}_{m \text { eff }}=\sum_{j} \mathbf{p}_{m j} N_{j},
$$

where $N_{j}$ is the number of metallic inclusions of sort $j$ inside the volume $V$ of the effective medium. On the other hand, the total dipole moment of the effective medium $\mathbf{p}_{m \text { eff }}$ is equal to the product of the magnetization $\mathbf{M}_{\text {eff }}$ and the volume $V$ of the medium:

$$
\mathbf{p}_{m \text { eff }}=\mathbf{M}_{\mathrm{eff}} V=\frac{\mu_{\mathrm{eff}}-1}{4 \pi} \mathbf{H}_{0} V .
$$

Inserting (6) and (8) in (7), we obtain an equation for the effective magnetic permeability (the degree of which is equal to the number of phases in the system):

$$
\mu_{\mathrm{eff}}=1-\frac{3}{2} \sum_{j} \frac{\mu_{\mathrm{eff}}\left[\xi_{j}\left(2 \mu_{j}+1\right)+(k a)^{2}\right]}{\xi_{j}\left(\mu_{\mathrm{eff}}-\mu_{j}\right)+(k a)^{2} \mu_{\mathrm{eff}}} \eta_{j} .
$$

Since the magnetic field outside the spherical particle is calculated within a quasistatic dipolar approximation, the particle radius must be small in comparison with the wavelength in the effective medium:

$$
\left|\frac{\omega}{c} \sqrt{\varepsilon_{\mathrm{eff}} \mu_{\mathrm{eff}}} a\right| \ll 1 .
$$

If the medium consists of metallic nonmagnetic particles with high conductivity $\left(4 \pi \sigma / \omega \gg \varepsilon^{\prime}\right)$ and void space, Eq. (9) reduces to a quadratic equation with two parameters: the ratio of the particle radius to the skin depth $a / \delta$ and the relative volume concentration of the particles $\eta$. Similar to the previous model (1)-(2), in the model described here the dependency of the imaginary part of the effective magnetic permeability on $a / \delta$ exhibits a maximum when the particle size and the skin depth are of the same order of magnitude.

Shown in Fig. 1 is the dependency of the real (a) and imaginary (b) parts of the effective magnetic permeability, $\mu_{\text {eff }}$, on the relative volume concentration of solid matter. In contrast to the model (1) - (2), in the proposed model the real part of $\mu_{\text {eff }}$ remains positive at high values 
of the particle concentration. The imaginary part exhibits a maximum at a particle concentration $\eta \approx 0.7$ (for $a / \delta \gtrsim 3)$. The decrease in the imaginary part of $\mu_{\text {eff }}$ at higher volume concentration of conductive inclusions is apparently due to the influence of a dense medium on the magnetic field in it. At $a / \delta \lesssim 3$ the imaginary part of $\mu_{\text {eff }}$ increases monotonically with increasing concentration.

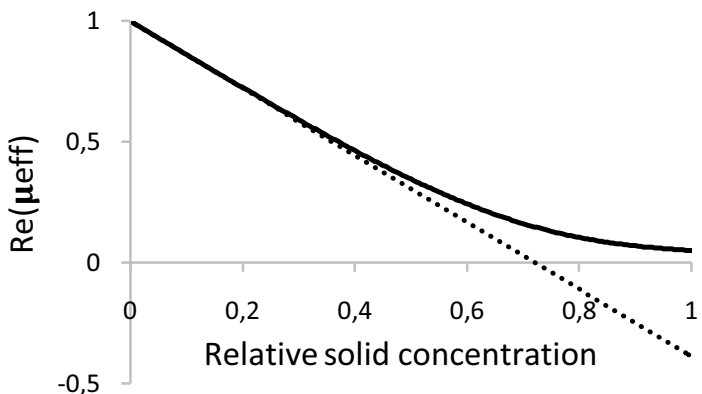

(a)

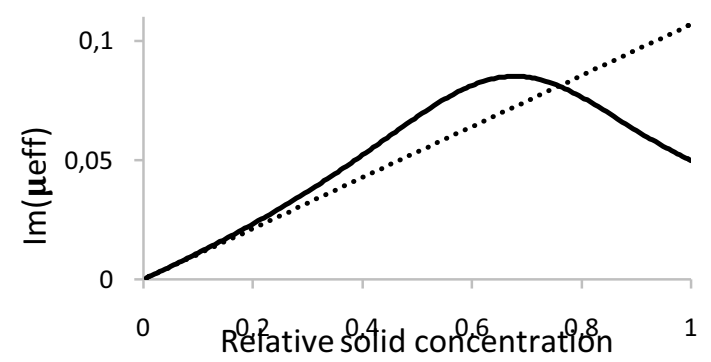

(b)

Fig. 1. The real (a) and imaginary (b) parts of the effective magnetic permeability of the metallic powder vs. the relative volume concentration of solid. The ratio of the particle radius to the skin depth is $a / \delta=20$. The solid line corresponds to the newly developed model (9), the dotted line corresponds to the previously used model (1) - (2)

As follows from Fig. 1, both models give the same result in the domain of low concentrations. With an increase in concentration, there are differences in the models in the value of $\mu_{\text {eff }}$ and in the character of the dependence of $\mu_{\text {eff }}$ on the concentration. When the volume fraction of particles tends to unity, the model (9) does not coincide with the case of a homogeneous metal, since it is assumed that the particles are electrically isolated from each other (e.g., by native oxide layers), and the eddy currents induced by the alternating magnetic field are localized inside small areas inside the medium.

Finally, it is necessary to determine the range of parameters in which the applicability condition (10) of the developed model is satisfied. The effective dielectric permittivity was calculated in the effective medium approximation that takes into account the inhomogeneous structure of the electromagnetic field inside metallic inclusions and the presence of insulating layers on metallic particles [3].
Shown in Fig. 2 is the dependency of the parameter $\left|\frac{\omega}{c} \sqrt{\varepsilon_{\text {eff }} \mu_{\text {eff }}} a\right|$ on the thickness of the insulating layer and the concentration of particles.

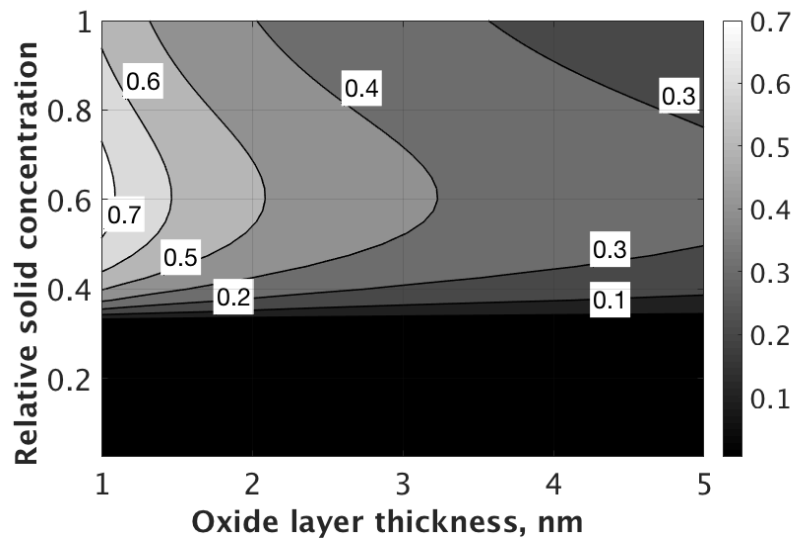

Fig. 2. Contour lines of the parameter $\left|\frac{\omega}{c} \sqrt{\varepsilon_{\mathrm{eff}} \mu_{\mathrm{eff}}} a\right|$ vs. the thickness of the insulating layer on particles (horizontal axis) and the relative volume concentration of particles (vertical axis). Copper powder, conductivity $\sigma=5.96 \cdot 10^{7} \mathrm{~S} / \mathrm{m}$, particle radius $a=10 \mu \mathrm{m}$, frequency $f=\omega / 2 \pi=24 \mathrm{GHz}$

It can be seen from Fig. 2 that for the given values of the conductivity and frequency the condition (10) is satisfied when the insulating layers on particles are not too thin. In particular, it is obviously satisfied at a concentration of metallic particles below the conductivity percolation threshold (0.33), since the absolute value of the effective permittivity of the medium differs little from unity in this case.

In conclusion, a model for the calculation of the effective magnetic permittivity of powder metal compacts has been developed. The proposed model makes it possible to determine the values of the real and imaginary parts of the magnetic permeability of metal powder compacts in the microwave range. The developed model can be used to calculate microwave absorption in dense compacts of metal particles insulated by oxide layers.

This research was supported by Russian Science Foundation, grant \# 17-19-01530.

\section{References}

1. Bergman, D. J., Stroud, D. Physical properties of macroscopically inhomogeneous media // Solid State Physics: Advances in Research and Applications, edited by H. Ehrenreich and D. Turnbull. New York: Academic Press. 1992. V. 46. P. 147-269.

2. Landau, L. D., Lifshits, L. P., Pitaevskii, E. M. Electrodynamics of Continuous Media. New York: Pergamon, 1960.

3. Rybakov, K. I., Semenov, V. E. Effective Microwave Dielectric Properties of Ensembles of Spherical Metal Particles // IEEE Transactions on Microwave Theory and Techniques. 2017. V. 65 , No. 5. P. $1479-1487$. 